\title{
A Treatment Recommender Clinical Decision Support System for Personalized Medicine: Method Development and Proof-of-concept for Drug Resistant Tuberculosis
}

Lennert Verboven ( $\sim$ lennert.verboven@uantwerpen.be)

University of Antwerp

Toon Calders

University of Antwerp

Steven Callens

Ghent University Hospital

John Black

University of Cape Town

Gary Maartens

University of Cape Town

Kelly E. Dooley

Johns Hopkins Medicine

Samantha Potgieter

University of the Free State

Robin M. Warren

Stellenbosch University

Kris Laukens

University of Antwerp

Annelies Van Rie

University of Antwerp

\section{Research Article}

Keywords: Clinical Decision Support System, treatment individualisation, machine learning

Posted Date: February 16th, 2021

DOl: https://doi.org/10.21203/rs.3.rs-199180/v1

License: (c) (1) This work is licensed under a Creative Commons Attribution 4.0 International License.

Read Full License 

1 Title: A treatment recommender clinical decision support system for personalized medicine:

2 method development and proof-of-concept for drug resistant tuberculosis

3 Authors: Lennert Verboven ${ }^{1,2}$, Toon Calders ${ }^{2}$, Steven Callens ${ }^{3}$, John Black ${ }^{4}$, Gary Maartens ${ }^{5}$,

4 Kelly E. Dooley ${ }^{6}$, Samantha Potgieter ${ }^{7}$, Robin M. Warren ${ }^{8}$, Kris Laukens ${ }^{2}$, Annelies Van Rie ${ }^{1}$

5 Affiliations:

6

1. Torch Consortium FAMPOP Faculty of Medicine and Health Sciences, University of Antwerp, Belgium

2. ADReM Data Lab, Department of Computer Science, University of Antwerp, Belgium

3. Department of Internal Medicine \& Infectious diseases, Ghent University Hospital, Ghent, Belgium

4. Department of Internal Medicine, University of Cape Town and Livingstone Hospital, Port Elizabeth, South Africa

5. Division of Clinical Pharmacology, Department of Medicine, University of Cape Town, South Africa

6. Divisions of Clinical Pharmacology and Infectious Diseases, Johns Hopkins University School of Medicine, Baltimore, MD USA

7. Division of Infectious Diseases, Department of Internal Medicine, Faculty of Health Sciences,

8. DSI-NRF Centre of Excellence for Biomedical Tuberculosis Research, SAMRC Centre for Tuberculosis Research, Division of Molecular Biology and Human Genetics, Stellenbosch University, Cape Town, 


\section{Background}

24 Individualised or precision medicine tailors care based on the patient's or pathogen's 25 genotypic and phenotypic characteristics. An automated Clinical Decision Support System 26 (CDSS) could help translate the genotypic and phenotypic characteristics into optimal

27 treatment and thus facilitate implementation of individualized treatment by less experienced 28 physicians.

\section{Methods}

30 We developed a hybrid knowledge- and data-driven treatment recommender CDSS.

31 Stakeholders and experts first define the knowledge base by identifying and quantifying drug

32 and regimen features for the prototype model input. In an iterative manner, feedback from

33 experts is harvested to generate model training datasets, machine learning methods are 34 applied to identify complex relations and patterns in the data, and model performance is 35 assessed by estimating the precision at one, mean reciprocal rank and mean average 36 precision. Once the model performance no longer iteratively increases, a validation dataset 37 is used to assess model overfitting.

\section{Results}

39 We applied the novel methodology to develop a treatment recommender CDSS for 40 individualized treatment of drug resistant tuberculosis as a proof of concept. Using input from

41 stakeholders and three rounds of expert feedback on a dataset of 355 patients with 129 42 unique drug resistance profiles, the model had a 95\% precision at 1 indicating that the highest 
43 ranked treatment regimen was considered appropriate by the experts in $95 \%$ of cases. Use of

44 a validation data set however suggested substantial model overfitting, with a reduction in 45 precision at 1 to $78 \%$.

\section{Conclusion}

47 Our novel and flexible hybrid knowledge- and data-driven treatment recommender CDSS is a

48 first step towards the automation of individualized treatment for personalized medicine.

49 Further research should assess its value in fields other than drug resistant tuberculosis, 50 develop solid statistical approaches to assess model performance, and evaluate their 51 accuracy in real-life clinical settings.

\section{KEYWORDS}

53 Clinical Decision Support System, treatment individualisation, machine learning

\section{INTRODUCTION}

56 Evidence-based medicine aims to integrate individual clinical experience with the best

57 available external scientific evidence to develop trustworthy clinical practice guidelines and 58 optimize clinical decision making $[1,2]$. Under the evidence-based medicine paradigm, the

59 clinician uses sound evidence to formulate the best therapeutic choice for their patient, most

60 often through a standardized public health approach where national or international

61 guidelines are implemented. More recently, personalised medicine has gained increasing

62 attention. Personalized or precision medicine tailors medical decisions to the individual

63 patient based on their predicted response to treatment in order to administer "therapy with 
64 the right drug at the right dose in the right patient" $[3,4]$. To implement personalized medicine, diagnostic tests are performed to determine the patient's and/or the pathogen's phenotypic and genetic characteristics. Integrating individual patient genomic information into a clinical decision is however challenging, especially for non-experts, given the rapid evolution in knowledge on the genotype-phenotype associations. The use of a clinical decision support system (CDSS) could facilitate the use of personalized medicine approaches by less experienced physicians and other health care workers at the time and location of patient care $[5,6]$.

CDSSs for guiding treatment decisions can either be data-driven or knowledge-driven. Knowledge-driven CDSSs use a rule-based system, implement guidelines developed by national or international organizations such as CDC and WHO, and operate at a rather coarse level and do not consider all available patient or pathogen information $[7,8]$. In contrast, data driven CDSSs use techniques such as machine learning and data mining aim to use all relevant data to learn complex relations and dynamics from past experience and reveal patterns in the data in order to assist with the complex decision making. For personalized medicine, datadriven CDSSs are attractive as data is increasingly being collected and stored [9-13].

Recommender systems use machine learning and data mining techniques to predict the preference a user would give to a specific item based on their preference history [14].

82 Recommender systems are mostly used to make personalized recommendations in ecommerce (e.g. Amazon), online media (e.g. Netflix), social media, and online news feeds [15]. Most recommender systems either use collaborative filtering, content-based filtering, or a combination of the two. Collaborative filtering recommender systems predict which items a 
user might like based on other similar users that watched similar items [16] and implicit (i.e.

87 a user watched a movie) or explicit (i.e. a user gave a 5-star rating) preferences by the user.

88 Content-based recommender systems predict which new items the user will like by learning

89 a classifier of the likes and dislikes of a user using the features associated with the items they

90 like. $[14,17]$. Data driven CDSSs and recommender systems are uncommon in clinical practice,

91 mostly due the perception that they are a 'black box' tool [6] with a decision process that

92 lacks transparency, even though transparent recommender systems exist $[18,19]$.

93 Crowdsourcing is a problem-solving model in which a large open group of actors try to 94 collectively solve a larger problem [20] with many applications such as street mapping 95 (OpenStreetMap; a collaborative effort of mappers contributing to create and maintain world 96 map data), and data science (Netflix Prize; an open competition to develop the best 97 collaborative filtering algorithm for Netflix [21]).

In this study we report the development of a fully automated hybrid knowledge- and datadriven CDSS to identify the optimal treatment regimen for individual patients and present the

100 personalized treatment of drug resistant tuberculosis as a use case to explain the 101 methodology and its potential application in global health.

102

\section{METHODS}

103 To ensure a transparent and standardized process, we adapted the multi-step approach for 104 the development of a decision aid [22] (Fig 1). In this methods section, we describe the 105 complete process of developing the treatment recommender CDSS. In the results section, we 
present the results of the application of this developed treatment recommender CDSS for the

107 individualized treatment of drug resistant tuberculosis.

\section{Defining the scope and assembling the expert panel}

109 After defining the purpose and target audience of the treatment recommender CDSS (scoping step), an expert panel was assembled (steering step). To ensure a multidisciplinary and

111 holistic approach, the panel represented expertise in pathogen genomics (knowledge of

112 genotype-phenotype associations), pharmacology (characteristics of the drugs, drug-drug 113 interactions, and synergy and antagonism between drugs), clinical experts, as well as health

114 system and health economic experts. In addition, patients were consulted to provide their 115 perspective (e.g. the impact of side effects on their quality of life).

\section{Assembling the knowledge base}

117 The knowledge base was developed in an iterative manner by combining review of published

118 literature, non-published data when gaps in the literature were identified, and consensus

119 building between experts using a standardized format for efficient expert feedback. First, the 120 key features of relevant individual drugs were determined. Next, the features of the

121 treatment regimen were established, hereafter referred to as regimen features, including the 122 number of effective drugs required and how drug features are aggregated into regimen 123 features. Third, data input requirements and decisions on user-friendly design of the 124 treatment recommender and communication of the recommendation were made.

\section{Model development}




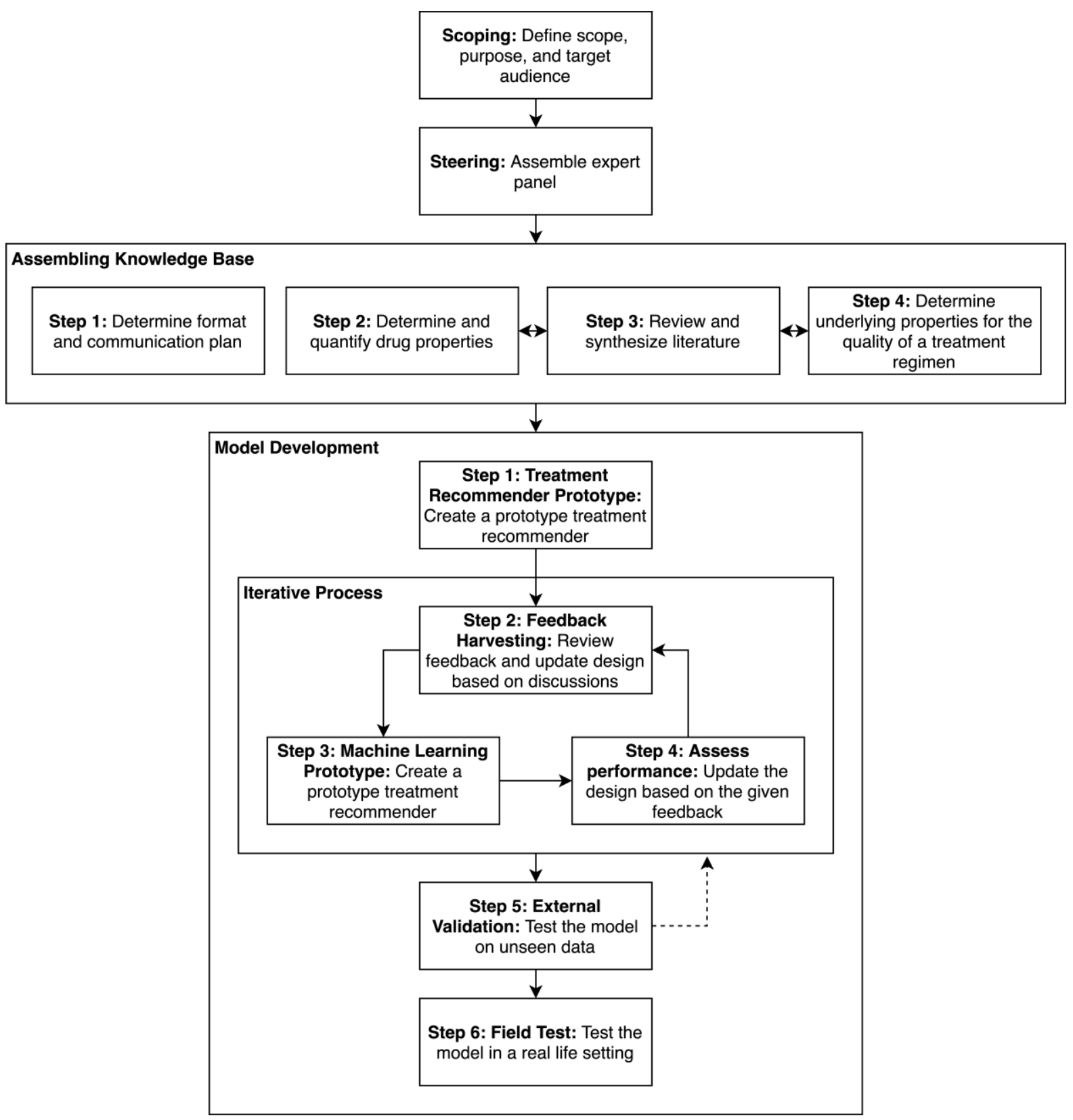

128 Development of the model consists of 6 steps (Figure 1). First, a prototype was developed to

129 rank all possibly valid treatment regimens for an individual patient. Second, expert feedback

130 was harvested on a sample of the top scoring regimens for patients that are representative

131 of the target population. Third, the expert feedback was used to develop a training dataset. 
132 Fourth, the model performance in recommending the optimal individual treatment regimen

133 was assessed. When the model performance was shown to be suboptimal, the rankings for

134 the patient-regimen pairs obtained by the random forest classifier were used in determining

135 the sampling for the next round of expert feedback harvesting. This process of machine

136 learning, expert data harvesting and assessment of model performance was repeated until

137 the model no longer substantially improved. Fifth, the final model was tested on a different

138 real-life clinical dataset to assess the degree of overfitting to the training data and verify that

139 the model is transferable to new data. Overfitting occurs when a model corresponds too

140 closely or exactly to the data on which it was trained, and may therefore underperform on

141 new and unseen data. The final step in the development process was a field test to assess the

142 effectiveness of the treatment recommender CDSS model for individualized treatment in

143 clinical trial participants. In the intervention arm, the minimum required patient information

144 and data on genomics information (treatment recommender CDSS input) was used by the

145 treatment recommender CDSS to proposes the optimal individualized treatment for that

146 patient (treatment recommender CDSS output). In the section below, we describe the first

147 three steps in greater detail.

148 Step 1: Developing the treatment recommender prototype

149 Based on the knowledge base assembled, the prototype computes a quality score for every

150 valid patient-regimen pair (Fig 2). A valid regimen is defined as a regimen that only contains

151 valid drugs, and valid drugs are defined as drugs that are effective (no resistance detected)

152 and can be included in the individualized treatment regimen because of absence of clinical

153 contraindications, drug stock outs, or country-specific drug licensing issues. 
154 The number of possible regimens $r$ for a given patient is:

155

$$
r=\left(\begin{array}{l}
n \\
k
\end{array}\right)=\frac{n !}{k !(n-k) !}
$$

157 where $n$ is the number of drugs available for a specific patient and $k$ is the number of effective 158 drugs required in a treatment regimen. If the number of drugs to be included in the 159 individualized treatment regimen can vary, then the total number of possible regimens for 160 each patient equals the sum of formula 1 for all possible values of $k$.

161 The total number of unique resistance profiles $r p$ for a disease is:

$$
r p=\sum_{k=0}^{d}\left(\begin{array}{l}
d \\
k
\end{array}\right)=2^{d}
$$

163 where $d$ is the total number of available drugs for the disease of interest.

164 In the prototype, all regimen features were normalised from 0 (bad) to 1 (good) on the patient

165 level, meaning that the entire 0 to 1 interval is used to represent each regimen feature even 166 in patients with few available drugs. A higher score can be interpreted as better for the patient 167 and the highest scoring regimen is assumed to be the best regimen for that patient. After 168 normalising all regimen features and inverting negative features, such that for all regimen 169 features a lower score means worse for the patient, the sum of all features equates to the 170 quality score for that regimen, which is then used to rank the regimens for individual patients.

\section{Step 2: Harvesting expert feedback}




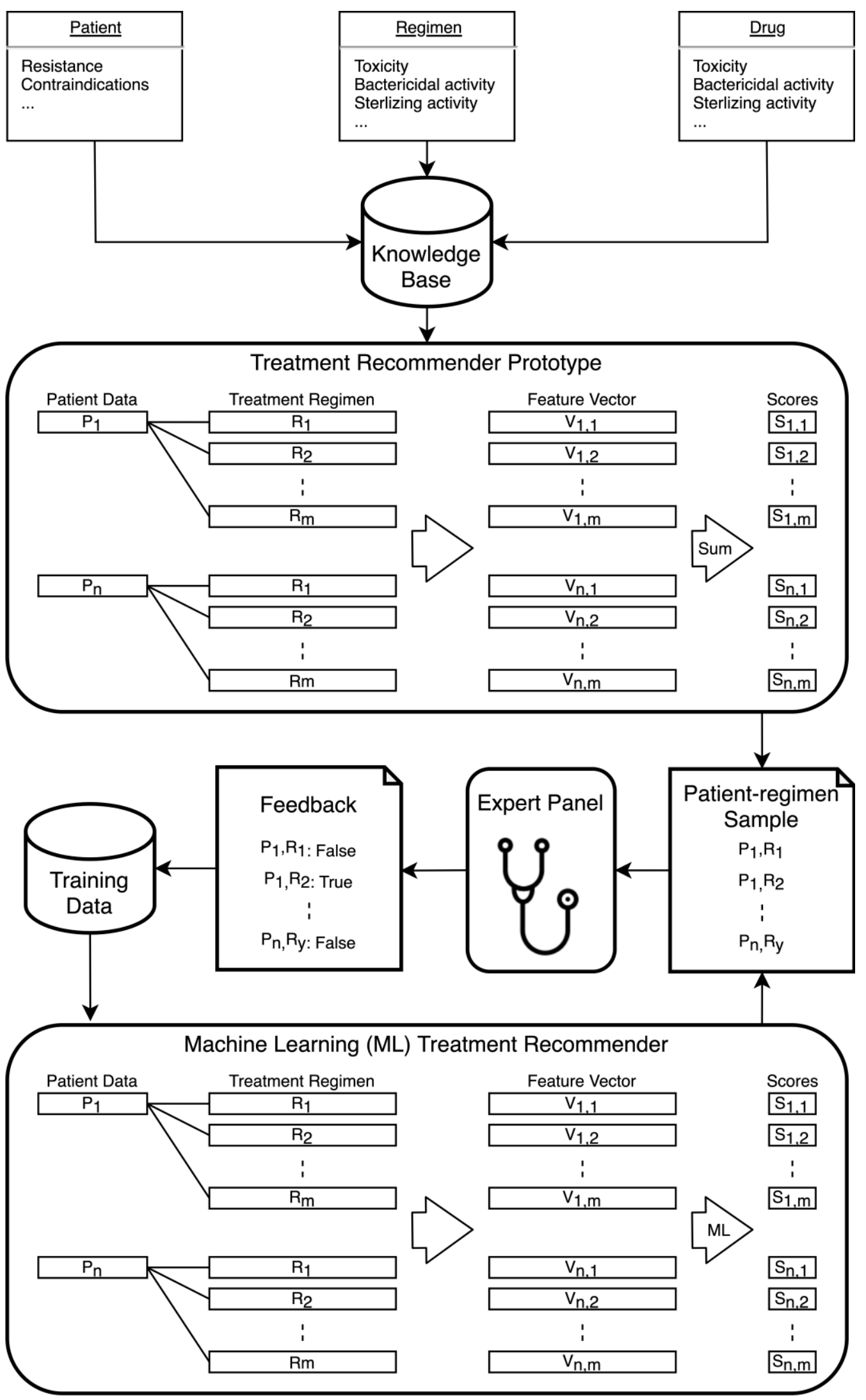


174 For each of the patients selected to represent the target patient population, a sample of the

175 top scoring regimens was reviewed by clinicians experienced in treating the condition of

176 interest (Figure 2). The number of cases reviewed was fixed and set to be large enough to

177 generate sufficient data to train the machine learning model but small enough so that the

178 experts were able and willing to carefully review every case presented. We sampled with

179 replacement to allow that multiple experts provide feedback on the same patient-regimen

180 pair, to allow that experts can provide feedback on the same patient-regimen pair multiple

181 times, and to ensure that a single expert cannot veto a top scoring regimen. The sampling

182 function randomly samples 1 regimen from the top 3 regimens (based on the ranking) for that patient. A regimen $i$ that has been sample before however has a probability $p_{i}$

$$
p_{i}=1-p_{\text {initial }}^{f_{i}}
$$

where $p_{\text {initial }}$ is the initial probability and $f_{i}$ is the amount of feedback already received for regimen $i$, to be removed from the top 3 making room for the next regimen in the ranking.

189 Parameter $p_{\text {initial }}$ can be tuned to make regimens more or less likely to be removed from the top 3. After tuning, we found $p_{\text {initial }}=0.2$ to give a good balance between resampling a regimen and allowing new, lower ranked regimens to be sampled. 


\section{Whole Genome Sequencing DST and Proposed Treatment Regimen}

\section{Resistant:}

Ethambutol

Ethionamide

Isoniazid

Isoniazid High Dose

Prothionamide

Rifabutin

Rifampicin

Streptomycin

Cycloserine

Pretomanid

Rifampicin High Dose

\section{Susceptible:}

Bedaquiline

Delamanid

Levofloxacin

Pyrazinamide

Moxifloxacin High Dose

Linezolid

Meropenem

Para-aminosalicylic acid

Amikacin

Moxifloxacin

Imipenem

Clofazimine

Terizidone
Proposed Regimen:

\begin{tabular}{l} 
Bedaquiline \\
\hline Delamanid \\
\hline Levofloxacin \\
\hline Pyrazinamide
\end{tabular}

MORE INFO

193

Would you prescribe the proposed treatment regimen?

No

YES

194 To harvest the experts' feedback on the regimen-patient pairs, we developed a secure web interface (Figure 3) that captured the feedback in a structured format. The web interface contained four components: list of drugs that are not valid for this patient (due to resistance, contraindications or not being available), list of valid drugs for the patient, and the recommended treatment regimen. Upon review of this information, the expert is asked whether he/she would prescribe this regimen for the individual patient. If the expert responded they would not prescribe the recommended regimen to the individual patient, they were asked why (open field) and were asked to list the regimen they would prescribe. The alternative regimen increased the number of patient-regimen pairs with positive feedback. The reasons for rejection were used to identify recurring topics that were then 
discussed with the experts. The outcome of these discussions led to additional literature review and possible addition, modification, or removal of drug or regimen features.

\section{Step 3: Data driven machine learning model development}

207 Our recommender model is different from standard recommender systems in that it does not use a patient's treatment history to propose a new treatment, instead our recommender model works by learning the underlying qualities of good treatments from other patients. The feedback given by the experts was stored in a database and served as training data for the

211 machine learning model. We used a random forest classifier to learn the importance of features and interactions between the features of a treatment regimen because random forest classifiers are robust against overfitting and easy to develop. An additional advantage of random forests is that they are constructed by having many decision trees vote and taking the consensus of these trees. The concept of a decision tree and the way they are used in random forests is very intuitive, making these more likely to be accepted by clinicians. Using

217 the normalised features, the model learns which regimen is good for a given patient, 218 accounting for the fact that not all options are available. The model is trained on the patientregimen-feedback pairs obtained through the expert feedback harvesting step and tries to predict the probability that a patient-regimen pair was deemed good by the experts (Figure 2). These probabilities are then again used to rank all patient-regimen pairs for a given

222 patient, with the highest ranked regimen being the optimal treatment for that patient.

223 We used a patient level leave-one-out cross validation strategy to predict the new ranking of 224 regimens for a given patient. In other words, when using the model to predict the ranking for 
patient $p$, we used the entire training data excluding all training data on $p$ to train the random

226 forest classifier.

\section{Step 4: Assessing model performance}

228 We used three patient level performance measures: Precision at 1 (P@1)which assesses the 229 highest ranked regimen, Mean Reciprocal Rank (MRR) which represents the average of how 230 high the first regimen is ranked over all patients by the model, and Mean Average Precision 231 (MAP) which takes the position of all appropriate regimens into account.

232 Precision at 1 is a performance parameter where precision at $\mathrm{N}$ is defined by equation 4 .

$$
P @ N=\frac{\# \text { good treatments in top } N}{\# \text { total treatments in top } N}
$$

234 P@1 is the fraction of patients for which the top ranked regimen is classified by the experts

235 as an appropriate regimen, with appropriate regimen defined as regimen the expert would

236 be willing to prescribe for that patient. When experts disagreed on the highest ranked

237 regimen, a majority voting was used to determine whether the regimen is appropriate.

238 The mean reciprocal rank is defined by equation 5 ,

$$
M R R=\frac{1}{|P|} \sum_{p}^{P} \frac{1}{\text { rank }_{p}}
$$

240 where $P$ is the set of patients and $\operatorname{rank}_{p}$ the position of the first regimen classified by the

241 experts as appropriate for patient $p$.

242 The mean average precision is defined by equation 6 , 


$$
M A P=\frac{1}{|P|} \sum_{p}^{P} \operatorname{Avg} P(p)
$$

244 where $\operatorname{Avg}(p)$ is defined in equation 7

$$
\operatorname{Avg}(p)=\frac{1}{\left|G T_{p}\right|} \sum_{i}^{T X_{p}}(P @ i \times \operatorname{rel}(i))
$$

246 where $G T_{p}$ is the set of appropriate treatments for patient $p, T X_{p}$ is the set of all valid

247 treatment regimens for patient $p$, and $\operatorname{rel}(i)$ indicates whether treatment $i$ is an appropriate

248 treatment for patient $p(\operatorname{rel}(i)=1)$, or an inappropriate treatment $(\operatorname{rel}(i)=0)$.

\section{RESULTS}

250 In this section, we present how the newly developed method was as a proof-of-concept, applied to develop a treatment recommender CDSS to guide the individualization of

252 treatment for Rifampicin Resistant Tuberculosis (RR-TB).

253 The scope and purpose of the development of a rifampicin resistant (RR)-TB treatment

254 recommender was defined as "improving RR-TB treatment outcomes by optimizing the 255 individualized treatment regimen in high TB burden resource limited settings". The primary 256 target audience were the clinicians treating patients with RR-TB in such setting.

257 A multidisciplinary steering group of experts was assembled by inviting clinicians with 258 experience in treating RR-TB, pharmacologists with expertise in TB drugs, molecular epidemiologists with expertise in interpretation of the genotype-phenotype associations

260 regarding drug resistance in Mycobacterium tuberculosis, health systems experts to assess integration of individualized treatment in routine care, and ex-RR-TB patients. 
262 The design phase started with a stakeholder meeting where all the members of the steering

263 group discussed the treatment recommender input parameters. Discussions focussed on

264 number of drugs needed in an effective treatment regimen, health system burden of

265 treatment monitoring, monitoring burden, drug toxicity, drug features, and clinical patient

266 characteristics, and genomic drug resistance profile. Uncertainties regarding the input

267 parameters identified during the meeting were resolved through literature search,

268 identification of unpublished data and iterative discussion until a consensus was reached on

269 all features to be included in the model. These discussions resulted in a set of 24 drugs that

270 are licensed for treatment of tuberculosis in South Africa, 9 drugs features, 18 regimen

271 features, and the consensus that 4 effective drugs need to be included in all individualized

272 regimens (Table 1). Based on these decisions, up to 10626 valid treatment regimens were

273 possible (Equation 1) for a patient without resistance. 


\begin{tabular}{|c|c|}
\hline Drugs & $\begin{array}{l}\text { Amikacin, Bedaquiline, Clofazimine, Cycloserine, Delamanid, Ethambutol, } \\
\text { Ethionamide, Imipenem, high or standard dose Isoniazid, Levofloxacin, } \\
\text { Linezolid, Meropenem, high or standard dose Moxifloxacin, Para- } \\
\text { aminosalicylic acid, Pretomanid, Prothionamide, Pyrazinamide, Rifabutin, } \\
\text { high or standard dose Rifampicin, Streptomycin, Terizidone, }\end{array}$ \\
\hline Drug features & $\begin{array}{l}\text { Route of administration, toxicity, QT prolongation, cost, early bactericidal } \\
\text { activity, bactericidal activity, sterilizing activity, mechanism of action, } \\
\text { propensity to acquire resistance }\end{array}$ \\
\hline Regimen features & 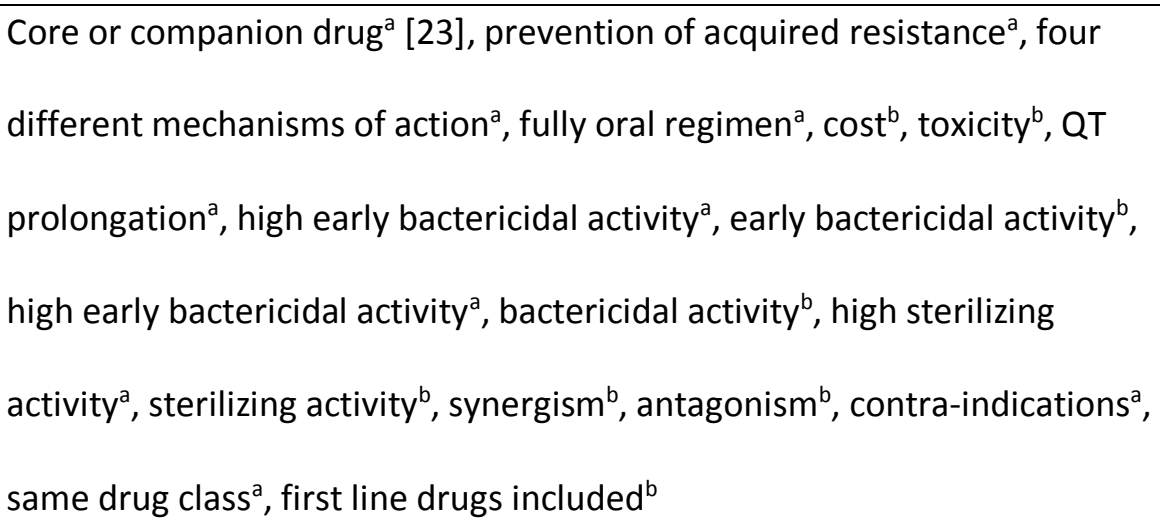 \\
\hline
\end{tabular}

277 We used a dataset that contained both clinical and whole genome sequencing data on 355

278 patients diagnosed with RR-TB in three provinces in South Africa. The whole genome 279 sequencing data represented 129 different drug resistance profiles. A group of 6 clinicians experienced in treatment of drug resistant tuberculosis were then asked to provide feedback on the recommended treatment using a structured online survey (Figure 3). 


\begin{tabular}{|c|c|c|c|c|c|c|}
\hline & $\begin{array}{l}\text { Number of } \\
\text { regimens } \\
\text { presented } \\
\text { to experts }\end{array}$ & $\begin{array}{l}\text { Total number } \\
\text { of } \\
\text { observations }^{\mathrm{a}}\end{array}$ & $\begin{array}{l}\text { Number of } \\
\text { participating } \\
\text { experts }\end{array}$ & $\begin{array}{l}\text { Precision } \\
\text { at } 1\end{array}$ & $\begin{array}{l}\text { Mean } \\
\text { average } \\
\text { precision }\end{array}$ & $\begin{array}{l}\text { Mean } \\
\text { reciprocal } \\
\text { rank }\end{array}$ \\
\hline Training round 1 & 479 & 855 & 5 & $89 \%$ & $53 \%$ & $90 \%$ \\
\hline Training round 2 & 445 & 719 & 6 & $95 \%$ & $69 \%$ & $97 \%$ \\
\hline Training round 3 & 360 & 607 & 6 & $95 \%$ & $72 \%$ & $95 \%$ \\
\hline Training round $1-3$ & 1284 & 2181 & & & & \\
\hline External Validation & 375 & 592 & 5 & $78 \%$ & $68 \%$ & $87 \%$ \\
\hline
\end{tabular}

Assessment of the performance of the prototype model showed precision at 1 of $89 \%$, mean average precision (MAP) of 53\% and mean reciprocal rank (MPR) of 90\% (Table 2). The written comments on regimens judged to be inappropriate were discussed with the expert group which resulted in modifications to the treatment recommender CDSS prototype. For example, the efficacy of the different drugs, which was initially quantified using a single feature was

292 changed to three features: early bactericidal activity, bactericidal activity and sterilizing activity. Using the feedback, a random forest classifier was used to identify complex relations 
and patterns in the data. Based on these results, the updated treatment recommender CDSS reclassified the order of valid treatment regimens from which a sample was drawn for a second round of feedback harvesting from the expert clinicians. The model had improved, with an increase in all three performance parameters. Precision at one increased from $89 \%$ to $95 \%$, MAP from $53 \%$ to $69 \%$ and MRR from $90 \%$ to $97 \%$. After three rounds, the performance no longer improved, with precision at 1 and MRR stabilizing at 95\% and MAP around $70 \%$.

301 For the external validation, another dataset consisting of 64 unique resistance profiles for

302 patients diagnosed with RR-TB in another province of was used. On this external validation set, the model performance was lower, with a precision at 1 of $78 \%$, MAP of $68 \%$ and MRR of $87 \%$ (table 2).

\section{DISCUSSION}

307 We developed a novel treatment recommender CDSS that combines a knowledge-driven approach using feedback harvesting methodology with a data-driven approach using machine

309 learning methods to automate the individualisation of treatment. The knowledge-driven component consists of input and feedback from stakeholders and experts. The data-driven

311 component consists of the machine learning methods to identify complex relations and 312 patterns.

313 Our approach is fundamentally different from standard knowledge-driven approaches to 314 CDSSs and offers several advantages for personalized medicine. Standard knowledge-driven 
systems implement clinical guidelines using if-then rules which allow limited treatment

316 individualisation [6] and offer little flexibility as they need a complete overhaul when new

317 drugs become available or new knowledge becomes available. In contrast, including new

318 drugs and incremental knowledge on existing drugs in our novel methodology is possible

319 without new model training through quantifying the features of the new drug or updating the

320 relevant drug features.

321 Our approach is also fundamentally different from the data-driven methods that have been

322 used for other CDSSs. Grasser et al. developed two therapy decision support systems that use

323 historical treatment data to individualise psoriasis treatment based on patient attributes and

324 past treatment attributes to predict the response to different therapies [6]. The main

325 limitations of this methodology are that it does not learn the underlying properties of optimal

326 treatment but bases decisions on which treatments have worked well in the past similar

327 patients. Consequently, this method suffers from concept drift where statistical properties of

328 data change over time due to the discovery of a new drug or new knowledge on drug features.

329 Our model is less subject to concept drift, as it is possible to assign features to a new drug or

330 update the features of the drugs when statistical properties of drug or regimen features

331 change.

332 Machine learning and artificial intelligence methods such as neural networks have been used

333 in the framework of personalized medicine to learn complex and nonlinear relationships

334 between prognostic features and an individual patient's risk of treatment failure [11]. This

335 approach requires a dataset with treatment outcomes. Because of the relative novelty of 336 personalized medicine, such datasets with treatment outcome only allow the model to learn 
the underlying properties of the current standard of care instead of novel individualized treatment regimens.

The application of our newly developed treatment CDSS methodology to the individualization

340 of treatment of RR-TB provided proof-of-principle by demonstrating that the novel approach

341 is well suited to guide a personalized medicine approach when multiple combination of drugs

342 are possible in one individual patient. Using the proposed method, a treatment recommender

343 CDSS for personalized treatment can be built in a relatively short period of time using a

344 combination of stakeholder input, published and unpublished evidence, and expert feedback.

345 By harvesting expert feedback on patient scenario's simulated from real-life data, we created

346 a minimal dataset consisting of diverse individual treatment regimens that are representative

347 for patient care. By allowing the experts to provide an alternative regimen when disagreeing

348 with the proposed regimen, the resulting dataset contained a wide variety of accepted and

349 rejected treatment regimens.

350 While our novel method has many strengths for the field of precision medicine, including the hybrid data- and knowledge-driven approach, the use of a structured 'crowdsourcing' approach with predetermined experts for treatment decision making research [24], and the high degree of flexibility, several limitations should be noted. First, given the novelty of our approach, there is no consensus method yet to assess the performance of the model and there are no clear decision boundaries on when to stop the iterative development process. The external validation of our model for drug resistant TB demonstrated substantial overfitting on the training data, suggesting that the current performance parameters may not accurately capture the model's performance. Using a more diverse set of patient profiles and 
repeating the development process on this new dataset in combination with the already

360 collected data is likely to reduce overfitting. As such the data now used for external validation

361 could be included as training data before bringing this model into clinical practice. Second,

362 the model was developed using a limited group of experts. It is unknown to which degree the

363 model development is dependent of the number and composition of the expert group. Use

364 of other performance parameters and evaluation of the model in clinical trials in different

365 regions of the world will be needed to determine its accuracy for real-life decision making in

366 a personalized medicine.

367 In conclusion, while novel and promising, our hybrid knowledge- and data-driven treatment

368 recommender CDSS for individualising treatment is an important first step in the

369 development of methods aimed to facilitate the widespread implementation of personalized

370 medicine. Further research should assess its value in fields other than drug resistant

371 tuberculosis, develop solid statistical approaches to assess model approaches, and evaluate

372 their accuracy in real-life clinical settings.

\section{LIST OF ABBREVIATIONS}

374 CDSS Clinical Decision Support System

375 MAP Mean Average Precision

$376 \quad$ MRR Mean Reciprocal Rank

377 P@1 Precision at 1

RR-TB Rifampicin Resistant Tuberculosis 


\section{Ethics approval and consent to participate}

381 The research was performed in accordance with the Declaration of Helsinki. Collection of the

382 clinical strains and the determination of the genotypic drug resistance profile was approved

383 by appropriate ethics committees. For the training dataset, ethical approval was obtained

384 from the Human Research Ethics Committee of the University of the Witwatersrand in South

385 Africa and the Institutional Review Board of the University of North Carolina at Chapel Hill in

386 the United States (reference numbers M111139 and 11-2273). Approval of study activities

387 was also obtained from relevant health authorities. Participants gave verbal informed consent

388 by phone (recorded). The Research Ethics Committee or Institutional Review Board of Human

389 Research Ethics Committee of the University of the Witwatersrand in South Africa (M111139)

390 and the Institutional Review Board of the University of North Carolina at Chapel Hill in the

391 United States (11-2273) waived the need of informed consent for patients who had died or

392 were lost to follow-up from TB care prior to study enrolment and could not be contacted

393 despite multiple attempts. For the validation dataset, ethical clearance was obtained from

394 Stellenbosch University Health Research Ethics Committee. This ethics approval (ref number

$395 \mathrm{N09/11/269)}$ has a waiver of informed consent. The study titled "a personalised

396 recommender system for whole genome sequencing-based individualised treatment for drug

397 resistant tuberculosis" reported in this manuscript was approved by the Ethics Committee of

398 the University Hospital Antwerp and the University of Antwerp (ref number 2107101). 
$400 \quad$ Not applicable

401 Availability of data and materials

402 The datasets analysed are available from the corresponding author on reasonable request.

403 Competing interests

404 Not applicable

$405 \quad$ Funding

406 Fonds Wetenschappelijk Onderzoek, 1S39119N

407 Fonds Wetenschappelijk Onderzoek, G0F8316N

408 U.S. Food and Drug Administration, R01FD005724

409 National Institute of Allergy and Infectious Diseases, K24AI150349

410 South African Medical Research Council

411 Department of Science and Innovation - National Research Foundation, South Africa

412 Authors' contributions

$413 \mathrm{LV}, \mathrm{AVR}, \mathrm{RW}$ and $\mathrm{KL}$ set up the concept of the study. $\mathrm{VV}, \mathrm{KL}, \mathrm{TC}$, and AVR developed the

414 methods. AVR was principal investigator of the study that collected the data used in the

415 training data set. RW is the principal investigator of the study that collected the data used in

416 the validation data set. SC, GM, SP, JB, and KD contributed as experts to the data. LV analysed

417 and interpreted the data. LV wrote the first draft; AVR and KL contributed substantially in 418 revising the manuscript. All authors read and approved the final version of the manuscript. 
420 We would like to thank everyone who helped make this research possible. Specifically, the 421 people who helped organise and participated in the expert panel meetings, namely Fransceca

422 Conradie, Heeran Makkan, Trevor Beattie, Margaretha De Vos, Anzaan Dippenaar, Marissa Klopper, Elise De Vos, Leen Rigouts, Noor Zakhura, and Makanda Goodman.

424

\section{REFERENCES}

426

427

1. Sackett, D.L., et al., Evidence based medicine: what it is and what it isn't. BMJ, 1996.

428

429

430

431

432

433

434

435

436

437

438

439

440

441

442

443

444

445

446

447

448

449

450

451

452 312(7023): p. 71-2.

2. Djulbegovic, B. and G.H. Guyatt, Progress in evidence-based medicine: a quarter century on. Lancet, 2017. 390(10092): p. 415-423.

3. Mancinelli, L., M. Cronin, and W. Sadee, Pharmacogenomics: the promise of personalized medicine. AAPS PharmSci, 2000. 2(1): p. E4.

4. Academy of Medical Sciences, Stratified, personalised or P4 medicine: a new direction for placing the patient at the centre of healthcare and health education. 2015.

5. Beeler, P.E., D.W. Bates, and B.L. Hug, Clinical decision support systems. Swiss Med Wkly, 2014. 144: p. w14073.

6. Grasser, F., et al., Therapy Decision Support Based on Recommender System Methods. J Healthc Eng, 2017. 2017: p. 8659460.

7. Berner, E.S. and T.J. La Lande, Overview of Clinical Decision Support Systems, in Clinical Decision Support Systems: Theory and Practice, E.S. Berner, Editor. 2007, Springer New York: New York, NY. p. 3-22.

8. Zhu, N., et al., A Decision Support System with Intelligent Recommendation for Multidisciplinary Medical Treatment. ACM Trans. Multimedia Comput. Commun. Appl., 2020. 16(1s): p. Article 33.

9. Wang, M., et al., Safe Medicine Recommendation via Medical Knowledge Graph

Embedding. arXiv 1710.05980, 2017.

10. Zhang, Y., et al., iDoctor: Personalized and professionalized medical recommendations based on hybrid matrix factorization. Future Generation Computer Systems, 2017. 66: p. 30-35.

11. Katzman, J.L., et al., DeepSurv: personalized treatment recommender system using a Cox proportional hazards deep neural network. BMC Med Res Methodol, 2018. 18(1): p. 24. 
12. Chen, Y., J.D. Elenee Argentinis, and G. Weber, IBM Watson: How Cognitive Computing Can Be Applied to Big Data Challenges in Life Sciences Research. Clin Ther, 2016. 38(4): p. 688-701.

13. Zauderer, M.G., et al., Piloting IBM Watson Oncology within Memorial Sloan Kettering's regional network. Journal of Clinical Oncology, 2014. 32(15_suppl): p. e17653-e17653.

14. Varga, E., Recommender Systems, in Practical Data Science with Python 3: Synthesizing Actionable Insights from Data. 2019, Apress: Berkeley, CA. p. 317-339.

15. Deldjoo, Y., et al., Recommender Systems Leveraging Multimedia Content. ACM Computing Surveys, 2020. 53(5): p. 1-38.

16. Breese, J.S., D. Heckerman, and C. Kadie, Empirical analysis of predictive algorithms for collaborative filtering, in Proceedings of the Fourteenth conference on Uncertainty in artificial intelligence. 1998, Morgan Kaufmann Publishers Inc.: Madison, Wisconsin. p. 43-52.

17. Ricci, F., L. Rokac, and B. Shapira, Recommender Systems Handbook. 2009: Springer.

18. Musto, C., et al., Linked open data-based explanations for transparent recommender systems. International Journal of Human-Computer Studies, 2019. 121: p. 93-107.

19. Ngo, T., J. Kunkel, and J. Ziegler, Exploring Mental Models for Transparent and Controllable Recommender Systems: A Qualitative Study, in Proceedings of the 28th ACM Conference on User Modeling, Adaptation and Personalization. 2020, Association for Computing Machinery: Genoa, Italy. p. 183-191.

20. Howe, J., The Rise of Crowdsourcing, in Wired. 2006.

21. Netflix. Netflix Prize. [cited 2021 02/02/2021]; Available from: https://netflixprize.com/.

22. Coulter, A., et al., A systematic development process for patient decision aids. BMC Med Inform Decis Mak, 2013. 13 Suppl 2: p. S2.

23. Van Deun, A., et al., Principles for constructing a tuberculosis treatment regimen: the role and definition of core and companion drugs. Int J Tuberc Lung Dis, 2018. 22(3): p. 239-245.

24. Wazny, K., Applications of crowdsourcing in health: an overview. J Glob Health, 2018. 8(1): p. 010502. 


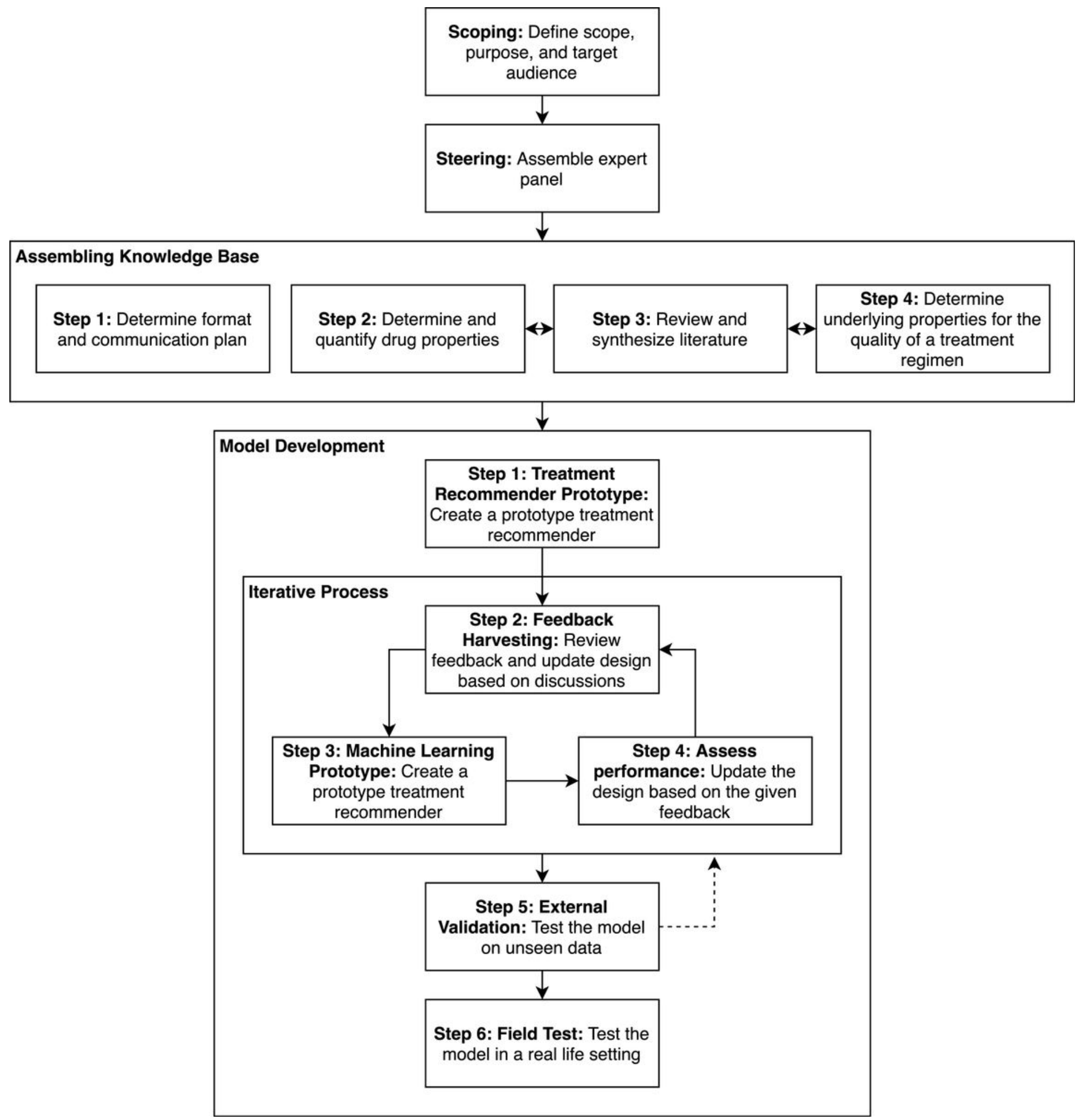

Figure 1

Steps in the development process of an automated clinical treatment recommendation system 


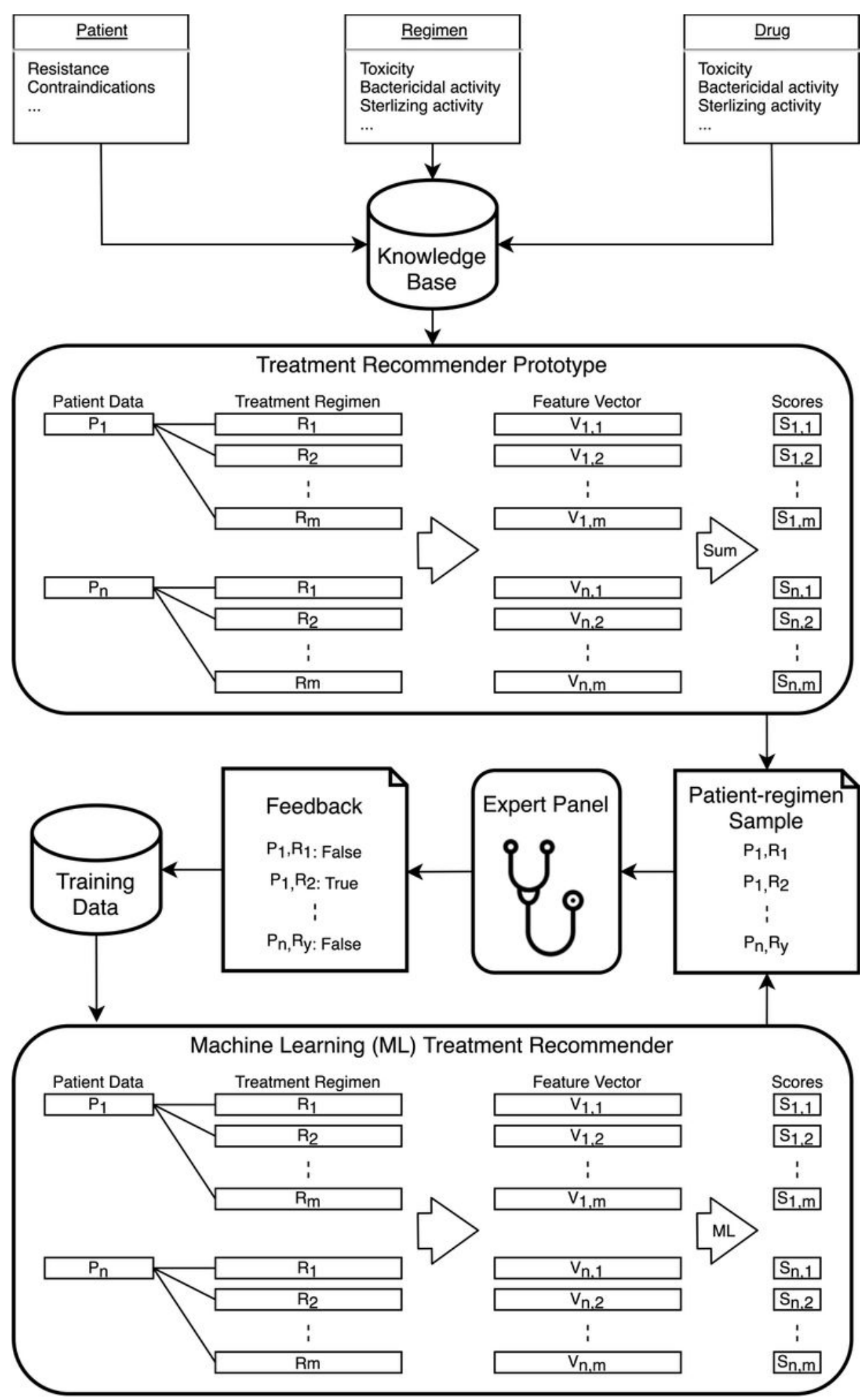

Figure 2

The model development flow 


\section{Whole Genome Sequencing DST and Proposed Treatment Regimen}

\section{Resistant:}

Ethambutol

Ethionamide

Isoniazid

Isoniazid High Dose

Prothionamide

Rifabutin

Rifampicin

Streptomycin

Cycloserine

Pretomanid

Rifampicin High Dose

\section{Susceptible:}

Bedaquiline

Delamanid

Levofloxacin

Pyrazinamide

Moxifloxacin High Dose

Linezolid

Meropenem

Para-aminosalicylic acid

Amikacin

Moxifloxacin

Imipenem

Clofazimine

Terizidone

\section{Proposed Regimen:}

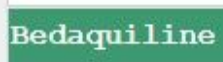

Delamanid

Levofloxacin

Pyrazinamide

MORE INFO

Would you prescribe the proposed treatment regimen?

No

YES

\section{Figure 3}

Layout of the web interface 